Trauma Berufskrankh 2014 · 16[Suppl 3]:263-273 DOI 10.1007/s10039-014-2074-6

Online publiziert: 15 . Mai 2014

(c) Springer-Verlag Berlin Heidelberg 2014

\author{
A. Kramer ${ }^{1,2} \cdot$ C.-D. Heidecke ${ }^{3,4}$ \\ ${ }^{1}$ Institut für Hygiene und Umweltmedizin, Universitätsmedizin Greifswald \\ ${ }^{2}$ Initiative Infektionsschutz, Berlin \\ ${ }^{3}$ Abteilung für Allgemeine, Viszeral-, Thorax- und Gefäßchirurgie, \\ Klinik für Chirurgie, Universitätsmedizin Greifswald \\ ${ }^{4}$ Chirurgische Arbeitsgemeinschaft für Qualität und Sicherheit in der Chirurgie (CAQS), \\ Deutsche Gesellschaft für Chirurgie, Berlin
}

\title{
Hygienevorschriften für die Operationsabteilung
}

Tradition ist. Diese Unterscheidung wird nachfolgend als Handlungshilfe getroffen.

Um die Sicherheit des Patienten im Rahmen operativer Eingriffe zu gewährleisten, sind neben der eindeutigen Identifizierung des Patienten und seines Operationsgebiets alle Maßnahmen zur Verhinderung postoperativer Wundinfektionen [SSI („surgical site infections“)] zu treffen. Das betrifft in erster Linie die direkt patientenbezogenen Maßnahmen, allerdings werden durch bauliche Voraussetzungen die Bemühungen der Infektionsprävention unterstützt.

Der Empfehlung „Safe surgery saves lives" der WHO (Weltgesundheitsorganisation; [129]) ist die grundsätzliche Aussage zu entnehmen, dass das Operationsteam alle zur SSI-Prävention als gesichert geltenden Maßnahmen konsequent umsetzen muss (sog. „best practice“) und die Ergebnisqualität mittels Surveillance zu evaluieren ist. Dabei werden als Schwerpunkte, weil besonders wichtig und häufig nicht konsequent umgesetzt, die Notwendigkeit der indikationsgerechten perioperativen Antibiotikaprophylaxe (PAP) innerhalb $1 \mathrm{~h}$ vor dem Schnitt (ist Bestandteil der "surgical safety checklist“ der WHO) und die sichere Aufbereitung von Medizinprodukten hervorgehoben.

Der Begriff der Hygienevorschrift lässt semantisch den Grad der Verbindlichkeit offen, d. h. was gesetzlich verbindlich, was evidenzbasierte Empfehlung, was Basishygiene, was kritisch zu hinterfragende

\section{Gesetzlich verbindliche Maßnahmen zur Prävention von SSI}

\section{IfSGuaÄndG}

Das IfSGuaÄndG (Gesetz zur Änderung des Infektionsschutzgesetzes und weiterer Gesetze; [23]) und die Landeshygieneverordnungen [68] bilden die Grundlage zur Gewährleistung der Struktur- und Prozessqualität als Voraussetzung zur erfolgreichen Prävention von HAI („,healthcareassociated infections") einschließlich SSI. Die Strukturqualität betrifft die Sicherstellung baulicher Anforderungen, die Einsetzung von Hygienefachpersonal (Krankenhaushygieniker und Hygienefachkräfte) mit der Maßgabe der Erfüllung der kapazitären Vorgaben bis 2016 und die Bestellung einer Hygienekommission.

Eckpfeiler der Qualitätssicherung sind

- der Aufbau eines mehrdimensionalen schnittstellenübergreifenden Qualitätsmanagements (QM) zur Realisierung der Praxis einer guten Krankenhaushygiene,

- die Realisierung von AntibiotikaStewardship-Programmen und

- die Surveillance von SSI einschließlich des Auftretens von Krankheitserregern mit speziellen Resistenzen und Multiresistenzen.
Für die Surveillance von SSI ist mindestens eine sog. Markeroperation auszuwählen. Wegen der Vergleichbarkeit kann es vorteilhaft sein, hierfür einen aseptischen Eingriff heranzuziehen. Entscheidend ist, dass sich für die Markeroperation eine repräsentative Anzahl ergibt. Zusätzlich sind Ausbrüche von HAI, d. h. $\geq 2$ gleichartige Infektionen, bei denen ein epidemischer Zusammenhang wahrscheinlich ist oder vermutet wird, innerhalb von $3 \mathrm{Ta}$ gen an das zuständige Gesundheitsamt zu melden [23]. Hinzu kommt die Einhaltung der 2009 eingeführten Meldepflicht eines MRSA-Nachweises (MRSA: methicillinresistenter Staphylococcus aureus) in Blut und Liquor.

Die Einsetzung von hygienebauftragten Ärzten und Pflegekräften („link nur$\mathrm{se}^{\text {") }}$ ist durch die Kommission für Krankenhaushygiene und Infektionsprävention beim Robert Koch-Institut (KRINKO) [78] geregelt. Im IfSGuaÄndG [23] wird zur Verbindlichkeit der Empfehlungen beider Kommissionen des Robert Koch-Instituts die wichtige Aussage getroffen, dass die Einhaltung des Standes der medizinischen Wissenschaft auf diesem Gebiet vermutet wird, wenn jeweils die veröffentlichten Empfehlungen der KRINKO und der Kommission Antiinfektiva, Resistenz und Therapie (ART) beachtet wurden. Gemäß der KRINKOEmpfehlung [78] sollte jedes Krankenhaus mindestens einen hygienebeauftragten Arzt berufen. In Behandlungszentren 


\section{Hygiene}

Tab. 1 Stellenwert wichtiger Maßnahmen zur Prävention von SSI

\begin{tabular}{|c|c|c|}
\hline Zeitpunkt & Maßnahme & Evidenz \\
\hline \multirow{23}{*}{$\begin{array}{l}\text { Prä-, peri- } \\
\text { operativ }\end{array}$} & Sanierung bestehender Infektionen vor elektiven Eingriffen & IA \\
\hline & Qualitätsgerechte Aufbereitung von MP & IA, IV \\
\hline & Aseptische Disziplin im OP & IB \\
\hline & Kurze präoperative Verweildauer & IB \\
\hline & Begrenzung der Personenanzahl im OP auf das erforderliche Maß & IB \\
\hline & $\begin{array}{l}\text { Screening und Dekolonisierung von Staphylococcus aureus bei } \\
\text { besonderer Gefährdung }\end{array}$ & II \\
\hline & MRSA-Screening bei Risikopatienten und Dekolonisierung & IB \\
\hline & Risikoadaptiertes Screening auf MRGN & II \\
\hline & Einstellung des Rauchens & IB \\
\hline & $\begin{array}{l}\text { Korrektur metabolischer Abweichungen (Serumalbumin, Glukose, Hb), } \\
\text { ggf. Gewichtsreduktion, jeweils bei elektiven Eingriffen }\end{array}$ & II \\
\hline & PAP bei gesicherter Indikation & IA \\
\hline & Antiseptisches Bad & III \\
\hline & Clipping oder keine Rasur & IA \\
\hline & Verbot künstlicher Fingernägel & IB/IV \\
\hline & Chirurgische Händedesinfektion & IB \\
\hline & Hautschutz der Hände & IV \\
\hline & Präoperative Hautantiseptik & IB \\
\hline & Hautversiegelung & III \\
\hline & Antiseptische Inzisionsfolie (indikationsabhängig) & II \\
\hline & Erregerdichte Operationsabdeckung & IB \\
\hline & Verzicht auf Darmreinigung in der Darmchirurgie & II \\
\hline & $\begin{array}{l}\text { Screening des Operationsteams beim Ausbruch von SSI durch } \\
\text { Staphylococcus aureus oder A-Streptokokken }\end{array}$ & IB \\
\hline & Sterile Operationshandschuhe, steriler Kittel, MNS, Haarschutz & IB \\
\hline \multirow{5}{*}{$\begin{array}{l}\text { Intra- } \\
\text { operativ }\end{array}$} & Vermeidung akzidenteller Hypothermie & IA \\
\hline & $\begin{array}{l}\text { Sterile Operationshandschuhe mit Wechsel bei intraoperativer } \\
\text { Beschädigung }\end{array}$ & IB \\
\hline & Antiseptisches Nahtmaterial (indikationsabhängig) & II \\
\hline & $\begin{array}{l}\text { Keine extrakorporale Implantatlagerung außerhalb Sterilverpackung } \\
\text { und außerhalb TAV }\end{array}$ & IB \\
\hline & TAV & III \\
\hline \multirow{4}{*}{$\begin{array}{l}\text { Post- } \\
\text { operativ }\end{array}$} & Desinfektion der relevanten Flächen und des relevanten Inventars im OP & IB \\
\hline & Aseptische Wundversorgung & IB \\
\hline & Strenge Indikationstellung für Drainagen & IB \\
\hline & Surveillance & IA/IV/ \\
\hline \multirow{5}{*}{$\begin{array}{l}\text { Rahmen- } \\
\text { bedingun- } \\
\text { gen }\end{array}$} & Operationstechnik und chirurgische Erfahrung & II \\
\hline & QM der Hygiene & IV \\
\hline & Einführung von SSI-Bündeln & IB \\
\hline & Fehleranalyse & IB \\
\hline & Evaluation der Hygiene durch Patienten & II \\
\hline \multicolumn{3}{|c|}{$\begin{array}{l}\text { Hb Hämoglobin, MNS Mund-Nasen-Schutz, MP Medizinprodukte, MRGN multiresistente gramnegative Stäb- } \\
\text { chenbakterien, MRSA methicillinresistenter Staphylococcus aureus, OP Operationssaal, PAP perioperative } \\
\text { Antibiotikaprophylaxe, QM Qualitätsmanagement, SSI, „surgical site infection“, TAV turbulenzarme Verdrän- } \\
\text { gungsströmung }\end{array}$} \\
\hline
\end{tabular}

mit mehreren organisatorisch getrennten Abteilungen und einem speziellen Risikoprofil für nosokomiale Infektionen, z. B. Chirurgie mit Intensivstation oder Neurochirurgie, soll jede Fachabteilung einen hygienebeauftragten Arzt bestellen.
Analoges gilt für ambulante Operationszentren.

Gemäß IfSGuaÄndG [23] haben die Leiter von Krankenhäusern, von Einrichtungen für ambulantes Operieren und weiterer hier nicht aufgeführter Einrichtungen sowie in einer Reihe von Bundes- ländern auch von Arztpraxen [68] sicherzustellen, dass die nach dem Stand der medizinischen Wissenschaft erforderlichen Maßnahmen getroffen werden, um HAI einschließlich SSI zu verhüten und die Weiterverbreitung von Krankheitserregern, insbesondere solcher mit Resistenzen, zu vermeiden. Dazu müssen die innerbetrieblichen Verfahrensweisen in Hygieneplänen festgelegt werden.

\section{KRINKO-Empfehlungen}

Folgende Empfehlungen sind für die Prävention von SSI besonders relevant:

- Prävention postoperativer Infektionen im Operationsgebiet [77],

- Anforderungen der Hygiene bei Operationen und anderen invasiven Eingriffen [72],

- Anforderungen der Hygiene beim ambulanten Operieren in Krankenhaus und Praxis [70],

- Händehygiene [73],

- Anforderungen an die Hygiene bei der Reinigung und Desinfektion von Flächen [76],

- Empfehlungen zur Surveillance [74, 75] mit Erläuterung [40],

- Empfehlung zur Prävention und Kontrolle von MRSA [71] sowie

- Hygienemaßnahmen bei Infektionen oder Besiedlung mit multiresistenten gramnegativen Bakterien (MRGN, [82]).

Den gesetzliche Rahmen für die Aufbereitung von Medizinprodukten (MP) schaffen das Gesetz und die Verordnung für Medizinprodukte [20] sowie die Medizinproduktebetreiberverordnung [22], während die detaillierten Anforderungen an die Hygiene bei der Aufbereitung von MP in der gemeinsame Empfehlung der KRINKO und des BfArM (Bundesinstitut für Arzneimittel und Medizinprodukte, [81]) verankert sind. In Studien wurde belegt, dass trotz Durchführung des Aufbereitungsprozesses Restkontaminationen feststellbar waren $[9,97]$. Eine Analyse in Südhessen ergab, dass die Voraussetzungen für die Durchführung des Aufbereitungsprozesses $\mathrm{z}$. T. nicht den gesetzlichen Bestimmungen entsprachen [114]. was sich mit anderen Berichten deckt [11, 49]. Als Hauptursachen für die Mängel 


\section{Tab. 2 Einteilung der KRINKO}

\begin{tabular}{|ll}
\hline Kategorie & Evidenzlage \\
\hline IA & $\begin{array}{l}\text { Die Empfehlung basiert auf gut konzipierten systematischen Übersichtsartikeln oder } \\
\text { einzelnen hochwertigen randomisierten kontrollierten Studien. }\end{array}$ \\
\hline IB & $\begin{array}{l}\text { Die Empfehlung basiert auf klinischen oder hochwertigen epidemiologischen Stu- } \\
\text { dien und strengen, plausiblen und nachvollziehbaren theoretischen Ableitungen. }\end{array}$ \\
\hline II & $\begin{array}{l}\text { Die Empfehlung basiert auf hinweisenden Studien/Untersuchungen und strengen, } \\
\text { plausiblen und nachvollziehbaren theoretischen Ableitungen. }\end{array}$ \\
\hline III & $\begin{array}{l}\text { Maßnahmen, über deren Wirksamkeit nur unzureichende oder widersprüchliche } \\
\text { Hinweise vorliegen, deshalb ist eine Empfehlung nicht möglich. }\end{array}$ \\
\hline IV & $\begin{array}{l}\text { Anforderungen, Maßnahmen und Verfahrensweisen, die durch allgemein geltende } \\
\text { Rechtsvorschriften zu beachten sind. }\end{array}$ \\
\hline KRINKO Kommission für Krankenhaushygiene und Infektionsprävention beim Robert Koch-Institut \\
\hline
\end{tabular}

erwiesen sich die hohen Kosten bei ordnungsgemäßer Durchführung der Aufbereitung, der untergeordnete Stellenwert, der der Aufbereitungsabteilung innerhalb der Gesundheitseinrichtung beigemessen wurde, und Defizite bei der behördlichen Überwachung [114]. Für die Aufbereitung von MP ergeben sich demnach folgende Konsequenzen:

- Für die Aufbereitung innerhalb der niedergelassenen Praxis besteht gemäß $§ 135$ a SGB (Sozialgesetzbuch) V die Verpflichtung zur Qualitätssicherung, es ist jedoch keine Zertifizierung erforderlich.

- Krankenhäuser, die für sich selbst und zusätzlich für andere Einrichtungen aufbereiten, müssen nicht zertifiziert und nicht beim DIMDI (Deutsches Institut für medizinische Dokumentation und Information) gemeldet sein [21]. Rechtlich ist die Situation des externen Aufbereiters aufgrund der Definition des sog. Inverkehrbringens von MP allerdings nicht eindeutig, sodass einige Juristen eine Zertifizierung empfehlen und viele Krankenhäuser sich daran orientieren.

- Die Aufbereitung von Kritisch-C-MP muss gemäß DIN EN ISO 13485/13488 [52, 53] durch eine von der zuständigen Behörde akkreditierte Stelle zertifiziert sein. Bei Auftragsvergabe ist die Aktualität der Dokumente zu überprüfen.

\section{Evidenzbasierte Empfehlungen}

Multibarrierenstrategie. Die gesicherten Maßnahmen zur Infektionspräven- tion werden sinnvollerweise aufeinander abgestimmt als sog. Multibarrierenstrategie umgesetzt. Dadurch gelingt es, die SSIRate im Behandlungsprozess zu minimieren [116, 131]. Voraussetzung dafür ist, dass die Bausteine der Multibarrierenstrategie im Konsens aller Beteiligten anhand der verfügbaren Evidenz festgelegt und ihre Einhaltung fortlaufend überprüft werden.

Ungeachtet der Evidenzstärke unterliegen die etablierten Standards zur SSIPrävention aufgrund neuer Erkenntnisse oder neuer Entwicklungen der fortlaufenden Evaluierung. Da sich die Studienlage seit der CDC-Leitlinie (CDC: „Center for Disease Control and Prevention") zur Prävention von SSI aus dem Jahr 1999 [88] und der KRINKO-Empfehlung aus dem Jahr 2007 [77] ständig erweiterte, sollen die Standards nachfolgend gemäß ihrer sich ergebenden Evidenz eingeordnet ( $($ Tab. 1) und bei veränderter Evidenzlage kurz kommentiert werden. Dabei werden über die KRINKO-Empfehlung [77] hinausgehende Quellen nur dann zitiert, wenn daraus eine veränderte Evidenz abgeleitet wurde. Zur Bewertung der Evidenzstärke wird die Einteilung der KRINKO (• Tab. 2, [80]) zugrunde gelegt.

\section{Prä-, perioperative Maßnahmen}

\section{Screening und Dekolonisierung von Sta-} phylococcus aureus. Nach Dekolonisierung mittels Screening identifizierter Träger von Staphylococcus aureus im Vestibulum nasi wurde die SSI-Rate bei Hüftimplantationen signifikant gesenkt [108]. Bei positivem Screeningergebnis auf Staphylococcus aureus war die Dekolonisierung kosteneffektiv (Level-II-Evidenz, [27]).
Trauma Berufskrankh 2014 • 16[Suppl 3]:263-273

DOI 10.1007/s10039-014-2074-6

(c) Springer-Verlag Berlin Heidelberg 2014

\section{A. Kramer $\cdot$ C.-D. Heidecke Hygienevorschriften für die Operationsabteilung}

\section{Zusammenfassung}

Hintergrund. Für Krankenhäuser und Arztpraxen besteht die gesetzliche Verpflichtung zur Qualitätssicherung. Der Aufbau eines Qualitätsmanagements zur Prävention von postoperativen Wundinfektionen [SSI (,surgical site infections")] ist eine Führungsaufgabe, die hohe Sachkenntnis und Konsequenz erfordert.

Präventionsmaßnahmen/Handlungsanweisungen. Im vorliegenden Beitrag werden die Maßnahmen zur Prävention von SSI in der Abfolge von prä-, peri-, intra- und postoperativen Maßnahmen einschließlich der für die Umsetzung erforderlichen Rahmenbedingungen einer Bewertung gemäß den vom Robert Koch-Institut empfohlenen Evidenzkategorien unterzogen, um daraus Handlungsanweisungen für die Praxis abzuleiten.

\section{Schlüsselwörter}

Hygiene · Asepsis · Postoperative Wundinfektionen · Prävention .

Qualitätssicherung

\section{Hygiene regulations for surgery departments}

\section{Abstract}

Background. For hospitals and medical practices there is a legal obligation to quality assurance. The development of a quality management system for the prevention of postoperative surgical site infections (SSI) is a management task that requires high skills and consistency.

Preventive measures and handling instructions. In this article the possibilities for the prevention of SSI in the sequence of preoperative, perioperative, intraoperative and postoperative measures including the conditions for implementation are evaluated in accordance with the evidence categories recommended by the Robert Koch Institute. Concluding instructions are derived for the surgical routine practice.

\section{Keywords}

Hygiene · Asepsis · Surgical site infection . Prevention · Quality assurance 
Selbst bei Screening und nasaler Dekolonisierung in Verbindung mit antiseptischer Körperwaschung bei allen chirurgisch aufgenommenen Patienten war der Einfluss auf die Herabsetzung der SSI-Rate signifikant $[15,109]$.

Dagegen war in der Kopf-Hals-Chirurgie durch Screening und Sanierung kein Einfluss nachweisbar [112].

Sofern die Folgen einer SSI durch Staphylococcus aureus besonders kritisch sind, z. B. bei Implantation einer HEP (Hüftendoprothese) oder KEP (Knieendoprothese), sollte das Screening mit nachfolgender Dekolonisierung erwogen werden. Für eine generelle Empfehlung ist die Evidenz derzeit noch nicht als ausreichend anzusehen.

MRSA-Screening. Es dient bei planbaren Operationen der Möglichkeit der präoperativen Dekolonisierung [3] bzw. bei nicht aufschiebbarer Operation im Fall der Indikation zur PAP der Auswahl des effektiven Antibiotikums und der gezielten Aufbereitung der Operationseinheit nach dem Eingriff [67].

Inzwischen werden auch für andere multiresistente Erreger (MRE) zunehmend Erfahrungen zu ihrem Vorkommen bestimmenden Risikofaktoren gesammelt. So ist die Gefahr des Auftretens von 3MRGN und 4MRGN (gegen 3 bzw. 4 Antiobiotikagruppen multiresistente gramnegative Stäbchenbakterien) bei positiver Anamnese, nach Kontakt mit bekanntermaßen mit 3- und 4MRGN kolonisierten oder infizierten Patienten und bei Patienten aus Ländern mit hoher Prävalenz gegeben [82].

Korrektur metabolischer Abweichungen bei elektiven Eingriffen. Bei Adipositas Grad II und III ist eine Gewichtsreduktion insbesondere für Eingriffe zu erwägen, bei denen der Operationserfolg von der Körpermasse beeinflusst wird [z. B. Hüft-TEP (TEP: Totalendoprothese), Knie-TEP, Narbenhernien; [77]].

Mangelernährung sollte präoperativ ausgeglichen werden. Grundsätzlich sollen Patienten bis zum Operationstag und so rasch wie möglich postoperativ beginnend enteral ernährt werden. Die Europäische Gesellschaft für klinische Ernährung und Metabolismus empfiehlt in ihren Leitlinien die präoperative Ernährung mangelernährter Patienten vor großen gastrointestinalen Eingriffen für die Dauer von 7 bis 14 Tagen.

Generell ist postoperativ keine Unterbrechung der Nahrungszufuhr erforderlich. Der frühzeitige orale bzw. enterale Kostaufbau führt gegenüber längerfristiger Nüchternheit zur Verminderung des SSI-Risikos [127].

Bei Diabetes mellitus sollte der Blutglukosespiegel prä- und postoperativ engmaschig kontrolliert werden mit Vermeidung von Werten $>200 \mathrm{mg} / \mathrm{dl}$ bzw. $>11,1 \mathrm{mmol} / \mathrm{l}$.

Eine präoperativ nachgewiesene Anämie sollte ausgeglichen werden [77].

Rauchen. Ein Verzicht ist mindestens 30 Tage vor dem Eingriff dringend nahezulegen, eine Karenz von 6 bis 8 Wochen vor elektiven Eingriffen reduziert die SSIRate signifikant [77].

PAP bei gesicherter Indikation. Entscheidend für den Beginn und die Dauer der Antibiotikagabe ist, dass ab dem Zeitpunkt des Hautschnitts für die Dauer der Operation ein wirksamer Blut- und Gewebespiegel gewährleistet ist. Für die Auswahl des Antibiotikums sind die Wirksamkeit gegen die häufigsten Wundinfektionserreger für die jeweilige Operation und die lokale Resistenzsituation entscheidend [77].

Clipping oder keine Rasur. Haare sind präoperativ nur bei operationstechnischer Notwendigkeit bevorzugt mittels Clipping zu entfernen [62].

Verbot künstlicher Fingernägel und Fingerringe. Die Bakteriendichte ist auf künstlichen Nägeln höher als auf natürlichen. Zugleich verleiten künstliche Nägel zur Vernachlässigung des Händewaschens und erhöhen die Perforationsgefahr für Schutzhandschuhe [47, 91, 107, $110,117]$. Als Reservoir für nosokomiale Erreger konnten wiederholt künstliche Nägel als Ursache für SSI-Ausbrüche identifiziert werden $[37,42,44,96,102$, 104, 106].

Das Tragen von Eheringen geht mit einer erhöhten Perforationshäufigkeit von Operationshandschuhen einher [99]. Da es für das Tragen von Ringen bisher keine Evidenz als Quelle von SSI gibt [2, 125], können Ringdosimeter toleriert werden, sofern sie von Seiten des Arbeitsschutzes vorgeschrieben sind. Für getragene Ringdosimeter ist es ausreichend, diese zur Desinfektion für $10 \mathrm{~min}$ in ein alkoholisches Flächendesinfektionsmittel einzulegen. Danach kann der Ring ohne Klarspülen mit Wasser nach Lufttrocknung erneut auf die desinfizierte Hand angelegt werden [61].

Chirurgische Händedesinfektion. Zur Gewährleistung der standardisierten Durchführung sollte das Vorgehen in einer SOP („standard operating procedure") festgelegt sein [63]. Für die Einwirkungszeit von $1,5 \mathrm{~min}$ ist folgendes Vorgehen effektiv: Zunächst werden beide Hände mit dem alkoholbasierten Präparaten vollständig benetzt $(10 \mathrm{~s})$. Im 2 . Schritt werden beide Unterarme benetzt (10 s). In der sich anschließenden Händedesinfektionsphase (70 s) ist darauf zu achten, dass Benetzungslücken verhindert werden, wobei das Hauptaugenmerk auf Fingerkuppen, Nagelfalze und Fingerzwischenräume zu legen ist [50].

Jodophore sind aus toxikologischen Gründen nicht mehr einzusetzen [12].

Hautschutz der Hände. Handschutz und -pflege sind eine berufliche Pflicht, weil eine geschädigte Haut nicht mehr sicher desinfizierbar ist [87], bereits kleinste Risse bzw. Mikrotraumen zum Erregerreservoir werden können $[38,83]$ und letztlich ein irritativ toxisches Kontaktekzem mit Berufsunfähigkeit entstehen kann. Um der Hautirritation vorzubeugen, müssen Hautschutz und -pflege systematisch und konsequent erfolgen. Dabei ist der präexpositionelle Hautschutz am wichtigsten und lässt sich nicht durch postexpositionelle Hautpflege ersetzen [13].

Anhand einer Fragebogenerhebung unter Chirurgen mit 1433 auswertbaren Datensätzen ergab sich ein erschreckendes Defizit in Bezug auf die Kenntnisse über den Unterschied zwischen Hautschutz und Hautpflege und die zeitliche Abfolge der Anwendung von Hautschutzund -pflegepräparaten [19]. Deshalb sind Hautschutz und -pflege in einem Hautschutzplan festzulegen. Bei der Auswahl 
der Präparate ist deren Eignung in Bezug auf den nachgewiesenen Nutzen zu berücksichtigen [69]. Außerdem muss gewährleistet sein, dass die Wirksamkeit der chirurgischen Händedesinfektion nicht beeinträchtigt wird [43].

Präoperative Hautantiseptik. Die Effektivität der präoperativen Hautantiseptik ist einer der Schlüsselpunkte für die Prävention von SSI. Mittel der Wahl sind Alkohole mit Zusatz remanenter Wirkstoffe, weil durch die Beigabe z. B. von Chlorhexidin oder Octenidin die Wirkung der Alkohole signifikant verbessert wird [29]. Allerdings wurde die Erregerzahl auf talgdrüsenreicher Haut, untersucht auf der Stirn, durch 70\% Propan-2-ol bei 10 min Benetzung nur um $1,1 \log _{10}$ reduziert. Durch Zusatz von 2\% Chlorhexidin zu 70\% Propan-2-ol stieg die Wirksamkeit auf $1,6 \log _{10}(\mathrm{p}<0,05)$ an, was in Anbetracht der durchschnittlichen Kolonisationsdichte auf talgdrüsenreicher Haut von $10^{5}-10^{6}$ [48] ebenfalls noch als unzureichend angesehen werden muss.

Durch die Entwicklung einer Vorrichtung zur assistierten Applikation mit Freigabe des Hautantiseptikums auf ein Schwämmchen (ChloraPrep ${ }^{\circledR}$ ) werden die wünschenswerte Standardisierung der präoperativen Hautantiseptik und zugleich eine höhere Effektivität erreicht. Gemäß Herstellerangabe wird die Haut im Operationsgebiet zunächst mit dem Schwämmchen $30 \mathrm{~s}$ mechanisch gewischt, danach soll sie 1,5 min benetzt bleiben. Alternativ ist das Vorgehen mit Tupfer und Kornzange mit gleicher Effektivität standardisierbar [121]. Der epidemiologische Nachweis für die Überlegenheit dieses Vorgehens im Vergleich zur mechanisch assistierten Applikation steht noch aus.

Insgesamt sind weitere Studien erforderlich, um die Wirksamkeit der Hautantiseptik auf $>3 \log _{10} \mathrm{zu}$ verbessern und dabei zugleich die Haarfollikel und Talgdrüsen zu erreichen.

Hautversiegelung. Um die auf der Haut nach Hautantiseptik verbleibende Mikroflora zu fixieren, wurde die Versiegelung der Haut nach Hautantiseptik eingeführt.

In einer retrospektiven nichtrandomisierten Studie in der Herzchirurgie wurde durch Anwendung des Wundversieglers
Integuseal ${ }^{\circledR}$ die SSI-Rate signifikant reduziert [31], was allerdings in einer nachfolgenden prospektiven Studie nicht bestätigt werden konnte [123]. Bei Gefäßoperationen an der unteren Extremität konnte in einer prospektiven, randomisierten, doppelblinden Studie dagegen wiederum eine signifikante Herabsetzung der SSIRate nachgewiesen werden [55]. Gestützt werden diese Befunde durch eine signifikante Minderung der wiedergewinnbaren Hautflora aus Inzisionswunden beim Schwein [128] bzw. beim Menschen nach Versiegelung [32, 118].

Antiseptische Inzisionsfolie. Bei nicht antiseptisch imprägnierter Inzisionsfolie steigt das SSI-Risiko signifikant, weshalb ihr Einsatz kontraindiziert ist [126]. Mit Jod imprägnierte Folie ist in vitro mikrobiozid wirksam [64]. Demzufolge kommt es auch bei ihrer Anwendung auf der Haut zu einer antiseptischen Wirkung unter der Inzisionsfolie, zugleich wird die bakterielle Wundkontamination verringert [64]. Aufgrund der geringen Effektstärke war die Reduktion der SSI-Rate jedoch nur bei großem Stichprobenumfang zu sichern [101].

Erregerdichte Operationsabdeckung. Nach präoperativer Antiseptik wird das Operationsfeld unter aseptischen Bedingungen mit als MP zertifizierten Abdeckmaterialien der Qualität „standard“ oder „high performance“ [8] steril abgedeckt.

Verzicht auf Darmreinigung in der Darmchirurgie. Sofern die präoperative Darmreinigung nicht aus operationstechnischer Sicht für erforderlich angesehen wird, ist sie als entbehrlich zu betrachten $[4,39,89,98]$.

Screening des Operationsteams. Wird im Rahmen der Surveillance eine Häufung von SSI durch Staphylococcus aureus oder Gruppe-A-Streptokokken auffällig, ist ein Screening hinsichtlich einer Kolonisation beim Operationsteam indiziert [77].
Intraoperative Maßnahmen

Vermeidung einer akzidentellen Hypothermie. Im Gegensatz zur therapeutisch oder protektiv erwünschten Hypothermie (z. B. bei bestimmten herzchirurgischen Eingriffen, Reanimation komatöser Patienten) ist eine akzidentelle Hypothermie zu vermeiden $[17,36,92]$. Allerdings konnte der präventive Einfluss auf die SSIRate als unabhängiger Faktor nicht durchgehend bestätigt werden [85], wobei die betreffende Studie methodische Grenzen aufwies. Aktives Erwärmen ist effektiver als passives Erwärmen, aber für besonders vulnerable Eingriffe kann die Kombination beider Möglichkeiten einschließlich präoperativer Erwärmung überlegen sein [95].

Mund-Nasen-Schutz, Haarschutz, steriler Kittel mit Wechsel vor jeder neuen Operation sowie bei sichtbarer Kontamination. Vor Betreten des OP (Operationssaal) werden Mund-Nasen-Schutz und Haarschutz angelegt, sofern die sterilen Instrumente bereits gerichtet sind, eine Operation demnächst beginnt oder durchgeführt wird. Die sterilen Operationshandschuhe werden nach dem Anlegen des sterilen Operationskittels übergestreift. Vor jeder neuen Operation muss die sterile Operationskleidung einschließlich Mund-Nasen- und Haarschutz gewechselt werden [77].

\section{Sterile Operationshandschuhe mit} Wechsel bei intraoperativer Beschädigung. Sterile Operationshandschuhe sind vor jedem direktem Kontakt mit nicht kolonisierten Bereichen des Körpers sowie im Umgang mit sterilen MP oder sterilem Material anzulegen. Dabei ist darauf zu achten, dass sie erst nach vollständiger Trocknung des Händedesinfektionsmittels anzuziehen sind, weil andernfalls das Perforationsrisiko steigt [105]. Durch unter dem Operationshandschuh getragene sterile Baumwollhandschuhe kann einem Feuchtigkeitsstau entgegengewirkt werden [14].

Es empfiehlt sich der Einsatz puderfreier Operationshandschuhe aus Naturlatex, da zurzeit von keinem anderen Material gleichwertige Eigenschaften hinsichtlich Tragekomfort, Passgenauigkeit, Grif- 
figkeit und mechanischer Belastbarkeit erreicht werden. Gepuderte Latexhandschuhe sind wegen des Allergisierungsrisikos untersagt [7]. Nachdem Talkum wegen des Auftretens von Talkumgranulomen nach Laparotomien in die Kritik geriet, wurde es durch Maisstärke ersetzt. Da auch hierfür die Bildung von Granulomen durch Kontamination des Operationsgebiets beschrieben wurde $[34,35,56,100$, 113], ist auch deren Einsatz abzulehnen. $\mathrm{Ob}$ das auch für in eine Emulsion eingebrachte Maisstärke (Biosorb ${ }^{\circledR}$ ) zutrifft, ist nicht untersucht. Da jedoch für Biosorb ${ }^{\star}$ kein signifikanter Einfluss auf die Handschweißmenge nachgewiesen werden konnte, ist dessen Einsatz als entbehrlich anzusehen [111].

Bei invasiven Eingriffen mit hoher Perforationsgefahr der Handschuhe wird das Tragen von 2 Paar Handschuhen empfohlen [77]. Alternativ sollten die Handschuhe bei viszeralchirurgischen Eingriffen durch den Operateur und den 1. Assistenten nach spätestens $90 \mathrm{~min}$, durch die weiteren Assistenten und Operationsschwestern nach 150 min gewechselt werden [ 45 , 103]. Bei intraoperativer Handschuhbeschädigung müssen 2 frische sterile Operationshandschuhe angelegt werden. $\mathrm{Zu}$ vor ist eine Händedesinfektion für die Dauer von 30 s durchzuführen [57, 58]. Ereignete sich die Perforation zum Ende der Operation, kann es ausreichend sein, einen frischen sterilen Handschuh über den perforierten Handschuh zu ziehen.

Bei Übertragungsrisiko für HIV („human immunodeficiency virus") oder Hepatitisviren (oder andere Infektionskrankheiten) vom Patienten auf das Personal sollte stets „double gloving“ mit Indikatorsystem gewählt werden. Alternativ kommen Operationshandschuhe mit zusätzlicher antimikrobieller Barriere in Betracht. Bei Nadelstichverletzung wurde dadurch der Bakterientransfer signifikant herabgesetzt [28], und es wurden $\geq 99 \%$ der eindringenden HCV (Hepatitis-C-Virus) und HIV inaktiviert [86]. Im Trageprozess wurde bei diesem Handschuhtyp die in den Handschuh freigesetzte Hautflora unabhängig von der Operationsdauer und der Art des Eingriffs signifikant herabgesetzt, sodass sich das Risiko für SSI reduzieren dürfte [6]. Nach Ablegen der Operationshandschuhe ist bei gegebener
Indikation eine hygienische Händedesinfektion durchzuführen, da die Hände durch unerkannte Leckagen oder Kontakt beim Abstreifen der Handschuhe kontaminiert worden sein können. Bei Eingriffen mit erhöhter Viruslast muss das ausgewählte Desinfektionsmittel, sofern es sich um unbehüllte Viren handelt, viruzid wirksam sein.

Extrakorporale aseptische Implantatlagerung. Befindet sich der Instrumententisch außerhalb des Lüftungsfelds des Laminar Air Flow, kommt es durch Erregeraufwirbelung aus der Umgebung zu dessen Kontamination [30].

Antiseptisches Nahtmaterial. Kommt es zur Biofilmbildung auf chirurgischem Nahtmaterial, sind die Mikroorganismen weitgehend vor der Wirtsabwehr geschützt und können entlang des Fadens tiefere Gewebeschichten erreichen. Ein Einfluss auf die SSI-Rate von antiseptisch imprägniertem Nahtmaterial wurde bei einer Reihe von Eingriffen nachgewiesen $[60,115]$. In einer Metanalyse wurde eine Herabsetzung der SSI-Rate um 30\% kalkuliert, mit konsistenten Ergebnissen bei abdominellen Eingriffen und sauberen oder sauber kontaminierten chirurgischen Wunden [124]. In einer in „Surgical Infections“ zum Druck akzeptierten erweiterten Metaanalyse mit 15 RCT (RCT: „randomized controlled trial“) und 4800 eingeschlossenen Patienten konnte die Effektivität des antiseptischen Nahtmaterials untermauert werden (Leaper, pers. Mitteilung). Die Autoren einer 2012 veröffentlichten Metaanalyse kamen noch zu dem Ergebnis, dass Nahtmaterial ohne signifikanten Einfluss auf die SSI-Rate sei [24]. In einer randomisierten Multicenterstudie bei kolorektalen Eingriffen war kein Effekt auf die SSI-Rate nachweisbar [10], was mit der im Darm dominierenden gramnegativen Flora im Zusammenhang stehen dürfte, da Triclosan unwirksam gegen Pseudomonas aeruginosa ist. Ebenso war bei gefäßchirurgischen Eingriffen an der unteren Extremität [120] und onkologischen Eingriffen im Kopf-Hals-Bereiche [25] kein Einfluss auf die SSI-Rate nachweisbar. Zur Eingrenzung des effektiven Indikationsbereichs bedarf es weiterführender RCT-Studien.
Keine extrakorporale Implantatlagerung außerhalb Sterilverpackung und TAV (turbulenzarme Verdrängungsströmung). Das ist v. a. dann essenziell, wenn sich der Instrumententisch außerhalb des Lüftungsfelds der RLTA (raumlufttechnische Anlage) befindet, weil es dann zu dessen Kontamination durch Erregeraufwirbelung aus der Umgebung kommt (s. oben, [30]).

Turbulenzarme Verdrängungsströmung. Während sich aus einigen Studien eine schwache Evidenz für den positiven Einfluss von Laminar Air Flow bei alloplastischem Hüft- und Kniegelenkersatz ableiten lässt, wurden diese Ergebnisse durch Analysen aus den Jahren 2008 und 2011 in Zweifel gestellt $[16,18,79]$. Allerdings wurden die SSI-Raten nicht im Rahmen kontrollierter Studien, sondern aus KISS-Daten (KISS: Krankenhausinfektionssurveillancesystem) generiert.

Zweifellos ist bei sog. kleinen operativen Eingriffen in einem Eingriffsraum und bei Eingriffen, für die die Risikobewertung ergibt, dass die in der Operationseinheit vorhandene Luftkoloniezahl der normalen Raumluft entsprechen kann, aus infektionsprophylaktischer Sicht keine RLTA erforderlich. Da jedoch durch eine leistungsfähige RLTA mit dynamischer TAV das Operationsfeld nahezu erreger- und partikelfrei gehalten wird, ist bei Hüft- und Kniegelenkimplantation durch TAV eine größere Sicherheit erreichbar. Hinzu kommt, dass sich die Investitionskosten zwischen Raumklasse Ib und la bei modernen Planungen nicht wesentlich unterscheiden. Schließlich besteht Konsens, dass zur Verhinderung implantatassoziierter Infektionen nach Hüftund Kniegelenkimplantation alle präventiven Möglichkeiten ausgeschöpft werden müssen, um eine Kontamination des alloplastischen Implantats mit nachfolgender Biofilmbildung und Infektion zu verhindern [67]. Voraussetzung ist die ausreichende Größe des Deckenfelds (etwa $518 \times 383 \mathrm{~cm}^{2}$ ), weil bei kleinem Ausmaß desselben $\left(380 \times 120 \mathrm{~cm}^{2}\right)$ die Effektivität der TAV durch Luftverwirbelungen signifikant herabgesetzt ist [30]. 


\section{Postoperative Maßnahmen}

\section{Desinfektion von relevanten Flächen} und Inventar im OP. Nach jeder Operation sind die patientennahen und die sichtbar kontaminierten Flächen sowie der begangene Fußbodenbereich im OP zu desinfizieren. Nach Betriebsende werden alle Fußbodenflächen und potenziell kontaminierte Flächen in allen Räumen der Operationsabteilung einer Wischdesinfektion unterzogen [77].

Aseptische Wundversorgung. Die primär verschlossene, nicht sezernierende Operationswunde wird am Ende des Eingriffs mit einer sterilen Wundauflage abgedeckt. Da nach 24 h keine Infektionsgefährdung mehr besteht, wird die Verwendung einer neuen Wundauflage vom Schutz vor mechanischer Belastung der Wunde bestimmt [77].

Strenge Indikationsstellung für Drainagen. Wunddrainagen sollen nicht routinemäßig, sondern nur bei Indikation und so kurzzeitig wie möglich eingesetzt werden. Offene Drainagen sind wegen des Infektionsrisikos nicht zu verwenden. Sofern Drainagen indiziert sind, sollen sie über eine separate Inzision gelegt werden [77].

Surveillance von SSI. Die Infektionssurveillance dient der Evaluierung der Maßnahmen der Primärprävention. Sie beinhaltet die fortlaufende Erfassung von SSI auf der Basis der Definitionen der US-amerikanischen CDC [88] mit Analyse und Interpretation der Inzidenz. Allein durch ihre Einführung wird bereits das Infektionsrisiko gesenkt [94]. Im KISS, Modul OP-KISS, können sog. häufig vorkommende bzw. besonders relevante Indikatoroperationen ausgewählt werden [41].

\section{Rahmenbedingungen}

Operationstechnik und chirurgische Erfahrung. Die Literatur ist wenig ergiebig bezüglich der Inzidenz von SSI und dem Einfluss chirurgischer Exzellenz bzw. Technik. Selbstverständlich ist ein atraumatischer Gewebeumgang wichtige Voraussetzung für eine primäre Wundhei- lung und somit für die Vermeidung von SSI [90]. Davon abgesehen gelten die allgemeingültigen Empfehlungen zur Vermeidung von Gewebeschädigungen.

In der Literatur finden sich keine Hinweise auf eine Korrelation von chirurgischer Exzellenz und SSI-Rate, aber auf eine solche mit chirurgischer Expertise. Bei Chirurgen mit hoher Fallzahl (also jenseits der Lernkurve) finden sich bei minimalinvasiver Rektumchirurgie deutlich weniger SSI als bei Kollegen, die sich noch in der Lernkurve befinden [54].

\section{Qualitätsmanagement der Hygie-} ne. Das IfSGuaÄndG [23] gewährleistet im Zusammenwirken mit den Landeshygieneverordnungen, den rechtsrelevanten Empfehlungen des Robert Koch-Instituts und den in Vorbereitung befindlichen Hygieneindikatoren des GBA (Gemeinsamer Bundesausschuss) die Rahmenbedingungen für die erfolgreiche Prävention von SSI. Im Fokus stehen die optimale Umsetzung der Primär-, Sekundärund Tertiärprävention mit Hilfe der Multibarrierenstrategie und deren fortlaufende Überprüfung z. B. mittels Checklisten, Hygienevisiten, Vor-Ort-Begehungen und In-Prozess-Kontrollen.

Für die Herstellung von Lebensmitteln ist das HACCP-Konzept (HACCP: „hazard analysis and critical control point“) seit langem Standard, um die Sicherheit des Verbrauchers auf dem höchstmöglich vertretbaren Sicherheitslevel zu gewährleisten. Die Etablierung eines QM der Krankenhaushygiene nach dem Vorbild des HACCP-Konzepts beinhaltet den Aufbau eines mehrdimensionalen Hygienekontrollsystems, das die Limitierungen einer nicht prozessual integrierten und nicht schnittstellenübergreifenden Betrachtung der Hygiene überwindet [51]. Schwerpunkte sind die Identifizierung von Risikopunkten und Infektionsreservoirs, die Beobachtung von Handlungsabläufen und deren Gefährdungsbeurteilung vor Ort durch Einbeziehung hygienisch-mikrobiologischer Untersuchungen sowie die Entwicklung von Konzepten der Infektionsprävention. Nicht die Einzelmaßnahme steht im Vordergrund, sondern die aufeinander abgestimmte Durchsetzung der anerkannten Maßnahmen zur Infektionsprävention einschließ- lich der Überwachung potenzieller Erregerreservoirs mit einem Warnsystem für kritische Pathogene.

Einführung von SSI-Bündeln. Es erwies sich als effektiv, besonders wichtige Maßnahmen zu einem sog. Maßnahmenbündel zusammenzufassen (sog. Bündelstrategie), die Bündel zu trainieren und deren Einhaltung in Form einer Selbstkontrolle mitttels Checkliste zu überwachen [26, 84, 119]. Durch das Eintrainieren mit nachfolgender Supervision wird die Compliance der Durchführung verbessert [1, 33]. Die Bündelstrategie begleitet die Kultur der Nulltoleranz, d. h. Nulltoleranz gegenüber Nichteinhaltung der gesicherten Maßnahmen zur Prävention von SSI, um deren Rate auf das erreichbare Minimum zu reduzieren.

Ein SSI-Bündel sollte in jedem Fall das risikoadaptierte präoperative MRSAScreening, die indikations- und zeitgerechte PAP, die standardisierte präoperative Hautantiseptik, die aseptische Disziplin des Operationsteams und die Surveillance umfassen. Clipping ist im Bündel entbehrlich, sofern die Rasierer konsequent eliminiert wurden.

Fehleranalyse. Wurden mehr als 2 Fehler bei der PAP (z. B. falscher Zeitpunkt, falsche Auswahl des Antibiotikums) analysiert, hatte das einen signifikanten Effekt auf die SSI-Rate [130]. Daraus kann im Umkehrschluss abgeleitet werden, dass durch eine Fehleranalyse Einfluss auf die SSI-Rate genommen werden kann. Ebenso war allein durch die Kontrolle der Einhaltung der PAP mittels Checkliste eine signifikante Senkung der SSI erreichbar [46].

Evaluation der Hygiene durch die $\mathrm{Pa}$ tienten. Nach Einführung des sog. Infektionspräventionschecks durch Kramer et al. [65] wurde das Hygieneverhalten der Ärzte höher bewertet als das des Pflegepersonals. Da üblicherweise von der umgekehrten Situation ausgegangen wird, ist $\mathrm{zu}$ vermuten, dass sich das Bewusstsein der Evaluierung durch die Patienten auf die Disziplin der Ärzte stärker als auf die des Pflegepersonals auswirkte [93]. 


\section{Basishygiene}

Die Voraussetzungen für eine effektive Händedesinfektion sind nur z. T. untersucht und leiten sich überwiegend aus der hygienischen Risikobewertung ab. Klinik und Praxis sind mit sauberen Händen und Fingernägeln $\mathrm{zu}$ betreten. Schmutzige Hände und Fingernägel (z. B. nach Gartenarbeit) sind bereits zu Hause zu säubern. Kommt es während der Tätigkeit zur Verschmutzung der Hände, sind ein Reinigungspräparat und wegen der Schonung der Haut nur im Bedarfsfall eine $\mathrm{Na}$ gelbürste zu benutzen. Kurzgeschnittene, mit den Fingerkuppen abschließende Fingernägel gewährleisten die gründliche Reinigung der subungualen Spatien und minimieren die Gefahr der Handschuhperforation an den Fingerkuppen.

Nagellack ist abzulehnen, nicht nur weil er die Sichtbeurteilung der Nägel verhindert, sondern weil bei 5 Tage altem Nagellack eine grenzwertig signifikante Unterlegenheit der Händedesinfektion im Vergleich zum Referenzverfahren ohne lackierte Nägel nachweisbar war. Obwohl dieser Einfluss bei frischem Nagellack nicht nachweisbar war, ist die Empfehlung, keinen Nagellack zu tragen, berechtigt, weil das Alter des Nagellacks und dessen Güte (Mikrorisse u. Ä.) in der Praxis nicht beurteilbar sind [5].

Hände und Fingernägel sollen beim Betreten des Operationstrakts sauber sein. Nägel und Nagelfalze sind bei Verschmutzung der subungualen Spatien mit weicher, thermisch desinfizierter Kunststoffbürste oder Einmalnagelbürste zu säubern. Hände und Unterarme sind wegen des Risikos der Wegbereitung von Hautirritationen und der damit verbundenen höheren Bakterienabgabe nicht mit einer Bürste zu behandeln [59, 77].

Vor operativen Eingriffen sollen keine Nagelbettverletzung oder entzündliche Prozesse an der Hand vorliegen [77]. Bei schwerer Onycholyse und Onychomykose des rechten Fingernagels mit gleichzeitigem subungualem Nachweis von Pseudomonas aeruginosa wurde ein Ausbruchgeschehen verursacht, obwohl chirurgische Latexhandschuhe getragen worden waren [91]. Auch bei Psoriasis mit Kolonisation durch Serratia marcescens wurde ein Ausbruch verursacht [122]. Unter sorgfältiger
Risikoabwägung erscheint es bei nichtentzündlichen Veränderungen bzw. kleinen Verletzungen im Bereich der Hand jedoch vertretbar, die Operation mit 2 übereinander gezogenen Paar Handschuhen, ggf. nach vorheriger Abdeckung mit antiseptischer Salbe und zusätzlichem Fingerling, durchzuführen [77]. Gegebenenfalls ist eine Vorstellung beim Betriebsarzt anzuraten.

Zur Operationsabteilung gehören ein oder mehrere Operationseinheiten, ggf. Haltezone und getrennte Aufwacheinheit, Personalschleuse mit unreiner und reiner Seite, Patientenzuführung, getrennte Ver- und Entsorgungsschleuse (Übergaberaum), Lagerräume, Aufenthaltsraum und Verwaltungsräume. Weitere Funktionen können auf Flächen statt in separaten Räumen realisiert werden. Für ambulante Patienten wird zusätzlich eine $\mathrm{Pa}$ tientenumkleide benötigt. Die nachfolgend zusammengefassten baulich-funktionellen Anforderungen beruhen auf dem hygienischen „risk assessment“ einschließlich der Überprüfung der theoretischen Plausibilität, weil eine systematische Überprüfung durch Studien methodisch nicht durchführbar ist. Folgende Anforderungen bzw. Raumforderungen wurden durch die KRINKO [72] erhoben und seinerzeit sogar mit Evidenzkategorien versehen:

- Abtrennung der Operationsabteilung vom übrigen Krankenhaus

- Patientenübergaberaum oder -fläche, alternativ Umbettung in Einleitungszone

- Innerhalb der Operationsabteilung keine Trennung der Flurwege (für Patienten, Personal, Güter usw.) mit Ausnahme ausgewiesener Sterilgutflure

- Ein Operationstisch pro Raum

- Als Räume ausgelegt: Personalschleuse, Aufenthaltsraum; unreiner Arbeitsraum, Entsorgungs- und Übergaberaum, Raum für Putzmittel - diese 3 Räume können kombiniert werden; ggf. Vorbereitungsraum für Instrumententische, Patientenumkleideraum, Aufwachraum

- Als Flächen ausreichend oder als Räume ausgelegt: Narkoseein- und -ausleitung entweder im OP oder in separaten Räumen/Flächen, Entsor- gung, Händewaschung und -desinfektion, Lager für saubere Geräte, Sterilgut u. a., Bettenabstellplatz, Übergabe reiner Güter, Notfalllabor, Dokumentations- und Verwaltungsaufgaben

- Keine Wasserarmaturen und Bodeneinläufe im $\mathrm{OP}$

\section{Kritisch zu hinterfragende Tradition}

In der KRINKO-Empfehlung [72] findet sich keine Aussage zur Notwendigkeit der baulichen Trennung zwischen aseptischem und septischem OP. Da es keine epidemiologische Evidenz für den Einfluss der baulichen Trennung auf die SSIRate gibt und auch das "risk assessment" nicht für die Notwendigkeit der Trennung spricht, ist die funktionelle Trennung als ausreichend anzusehen [66]. Sie kann durch folgende Maßnahmen erreicht werden:

- Ein Operationsprogramm mit aseptischen Operationen zu Beginn und septischen Operationen am Ende des Programms kann organisatorisch vorteilhaft sein, ist jedoch nicht zwingend erforderlich.

- Falls Mischströmung anstatt TAV-Decke: Schlussdesinfektion nach septischer Operation und bei bekannter Kolonisation mit MRE, OP bis zum Abschluss der Desinfektion als septisch kennzeichnen

- Wechsel der Reinigungsutensilien und Bereichskleidung des Reinigungspersonals nach septischer Operation

- Ausschleusung des Operationsteams mit Entsorgung der Operationsschutzkleidung innerhalb der Operationseinheit und Schuhwechsel nach septischer Operation mit erneuter Einschleusung

- Für Anästhesieteam und Springer: Wechsel der Bereichskleidung nach septischer Operation

Bei entsprechend großer Anzahl septischer Patienten kann es selbstverständlich logistisch vorteilhaft sein, einen separaten septischen OP vorzuhalten. 


\section{Fazit für die Praxis}

- Durch Einhaltung der gesicherten Standards zur Infektionsprävention lässt sich die Rate von SSI auf das erreichbare Minimum senken.

- Aufgrund des endogenen Infektionsrisikos ist die Nulltoleranz nur für Handlungslücken, nicht aber für SSI erreichbar.

- Die klare Regelung der Arbeitsabläufe prä-, intra- und postoperativ, die Einführung eines SSI-Bündels mit Training, Supervision und Selbstbewertung mittels Checkliste und die Surveillance von SSI bilden die Eckpfeiler der Praxis einer guten Krankenhaushygiene.

- Die Einbeziehung der Patienten in die Evaluation ist eine weitere Möglichkeit zur Umsetzung der Philosophie der Nulltoleranz gegenüber Hygienemängeln.

- Eine Sicherheitskultur ist nur dann erfolgreich zu etablieren, wenn Hygiene zur Chefsache erklärt wird!

\section{Korrespondenzadresse}

\section{Prof. Dr. A. Kramer}

Institut für Hygiene und Umweltmedizin, Universitätsmedizin Greifswald, Walther Rathenau Straße 49a, 17489 Greifswald kramer@uni-greifswald.de

\section{Einhaltung ethischer Richtlinien}

Interessenkonflikt. A. Kramer gibt Vortragstätigkeiten für Ethicon, 3M, Schülke und B. Bremen an. C.-D. Heidecke gibt Vortragstätigkeiten für Johnson \& Johnson, Bayer Vital und Applied Medical an.

Dieser Beitrag beinhaltet keine Studien an Menschen oder Tieren.

The supplement containing this article is not sponsored by industry.

\section{Literatur}

1. Aboelela SW, Stone PW, Larson EL (2007) Effectiveness of bundled behavioural interventions to control healthcare-associated infections: a systematic review of the literature. J Hosp Infect 66:101-108

2. Al-Allak A, Sarasin S, Key S, Morris-Stiff G (2008) Wedding rings are not a significant source of bacterial contamination following surgical scrubbing. Ann R Coll Surg Engl 90:133-135
3. Ammerlaan HSM, Kluytmans JAJW, Wertheim HFL et al (2009) Eradication of methicillin-resistant Staphylococcus aureus carriage: a systematic review. Clin Infect Dis 48:922-930

4. Anthony T, Murray BW, Sum-Ping JT et al (2011) Evaluating an evidence-based bundle for preventing surgical site infection: a randomized trial. Arch Surg 146:263-269

5. Assadian O, Benkhai H, Blacky A et al (2012) Einfluss von Nagellack auf die Effektivität der Händehygiene. 11. Kongress für Krankenhaushygiene, DGKH, 25.-28.03.2012, Berlin

6. Assadian O, Kramer A, Ouriel K et al (2013) Suppression of surgeons' bacterial hand flora during surgical procedures with a new antimicrobial surgical glove. Surg Infect (Larchmt) 15:43-49. DOI 10.1089/sur.2012.230

7. Ausschuss für Gefahrstoffe (AGS) (2011) Gefährdung durch Hautkontakt, Ermittlung - Beurteilung - Maßnahmen. TRGS - Technische Regeln für Gefahrstoffe 401. GMBI 9:175

8. AWMF (2010) OP-Kleidung und Patientenabdeckung. AWMF-Leitlinien-Register Nr. 029/012. Hyg Med 35:367-369

9. Azizi J, Anderson SG, Murphy S, Pryce S (2012) Uphill grime: process improvement in surgical instrument cleaning. AORN J 96:152-162

10. Baracs J, Huszar O, Sajjadi SG, Horváth OP (2011) Surgical site infections after abdominal closure in colorectal surgery using tridosan-coated absorbable suture (pds plus) vs. uncoated sutures (pds ii): a randomized multicenter study. Surg Infect (Larchmt) 12:483-489

11. Barion J (2011) Inspektion der Aufbereitung. Medizinprod J 18:110-113

12. Below H, Brauer VFH, Kramer A (2007) lodresorption bei antiseptischer Anwendung von lodophoren und Schlussfolgerungen zur Risikobewertung. GMS Krankenhhyg Interdiszip 2:Doc41

13. Berndt U, Gabard B, Schliemann-Willers S et al (2002) Integrated skin protection from work place irritants: a new model for efficacy assessment. Exogen Dermatol 1:45-48

14. Berufsgenossenschaft für Gesundheitsdienst und Wohlfahrtspflege (BGW) (2013) Hauptsache Hautschutz, Erstveröffentlichung 08/2007, Stand 05/2013. BGW, Hamburg. http://www.bgw-online.de/SharedDocs/Downloads/DE/Medientypen/bgw-themen/M650_Hauptsache\%20Hautschutz_Download.pdf?_blob=publicationFile. Zugegriffen: 11.03.2014

15. Bode LG, Kluytmans JA, Wertheim HF et al (2010) Preventing surgical-site infections in nasal carriers of Staphylococcus aureus. N Engl J Med 362:9-17

16. Brandt C, Hott U, Sohr D et al (2008) Operating room ventilation with laminar airflow shows no protective effect on the surgical site infection rate in orthopedic and abdominal surgery. Ann Surg 248:695-700

17. Brandt S, Mühlsteff J, Imhoff M (2013) VDE-Positionspapier Akzidentelle Hypothermie - Diagnose, Prävention und Therapie. Deutsche Gesellschaft für Biomedizinische Technik im VDE (Verband der Elektrotechnik und Elektronik). VDE, Frankfurt am Main. http://www.vde.com/de/fg/ DGBMT/Arbeitsgebiete/Fachausschuesse/puw/ Documents/VDE_PP_Ak.-Hypothermie-_RZ_ Web.pdf. Zugegriffen: 11.03.2014

18. Breier AC, Brandt C, Sohr D et al (2011) Laminar airflow ceiling size: no impact on infection rates following hip and knee prosthesis. Infect Control Hosp Epidemiol 32:1097-1102
19. Brune L, Harnoss JC, Ansorg J et al (2014) Ergebnisse einer Fragebogenerhebung des BDC zum Stellenwert von Hautschutz und Hautpflege bei chirurgischem Personal. Handschutz und -pflege als berufliche Pflicht. Passion Chir 4:2-6

20. Bundesregierung Deutschland (2002) Gesetz über Medizinprodukte (Medizinproduktegesetz, MPG) in der Fassung der Bekanntmachung vom 7. August 2002; zuletzt geändert durch Art. $12 \mathrm{G}$ vom 24.7.2010. Bundesregierung Deutschland, Berlin. http://www.gesetze-im-internet. de/bundesrecht/mpg/gesamt.pdf. Zugegriffen: 11.03.2014

21. Bundesregierung Deutschland (2003) Bekanntmachung gemäß § 2 Abs. 3 der Verordnung über das datenbankgestützte Informationssystem des Deutschen Instituts für Medizinische Dokumentation und Information (DIMDI-Verordnung) zu den Modalitäten der Durchführung der Anzeigen nach $\S$ 20, 24, 25 und 30 des Medizinproduktegesetzes. BAnz 118:13.869

22. Bundesregierung Deutschland (2009) Medizinprodukte-Betreiberverordnung (MPBetreibV). Verordnung über das Errichten, Betreiben und Anwenden von Medizinprodukten. I.d.F. Bekanntmachung vom 21.08.2002; BGBL 2002; I:3.396, zuletzt geändert durch Artikel 4 des Gesetzes vom 29. Juli 2009. BGBL 1:2326. http:// www.gesetze-im-internet.de/bundesrecht/ mpbetreibv/gesamt.pdf. Zugegriffen: 11.03.2014

23. Bundesregierung Deutschland (2011) Gesetz zur Änderung des Infektionsschutzgesetzes und weiterer Gesetze (IfSGuaÄndG) vom 04.08.2011. BGBL 1:1622

24. Chang WK, Srinivasa S, Morton R, Hill AG (2012) Triclosan-impregnated sutures to decrease surgical site infections: systematic review and metaanalysis of randomized trials. Ann Surg 255:854859

25. Chen SY, Chen TM, Dai NT et al (2011) Do antibacterial-coated sutures reduce wound infection in head and neck cancer reconstruction? Eur J Surg Oncol 37:300-304

26. Corcoran S, Jackson V, Coulter-Smith S et al (2013) Surgical site infection after cesarean section: implementing 3 changes to improve the quality of patient care. Am J Infect Control 41:12581263

27. Courville XF, Tomek IM, Kirkland KB et al (2012) Cost-effectiveness of preoperative nasal mupirocin treatment in preventing surgical site infection in patients undergoing total hip and knee arthroplasty: a cost-effectiveness analysis. Infect Control Hosp Epidemiol 33:152-159

28. Daeschlein G, Kramer A, Arnold A et al (2011) Evaluation of an innovative antimicrobial surgical glove technology to reduce the risk of microbial passage following intraoperative perforation. Am J Infect Control 39:98-103

29. Darouiche RO, Wall MJ, Itani KM et al (2010) Chlorhexidine-alcohol versus povidone-iodine for surgical-site antisepsis. N Engl J Med 362:1826

30. Diab-Elschahawi M, Berger J, Blacky A et al (2011) Impact of different-sized laminar air flow versus no laminar air flow on bacterial counts in the operating room during orthopedic surgery. Am J Infect Control 39:25-29

31. Dohmen PM, Gabbieri D, Weymann A et al (2011) A retrospective non-randomized study on the impact of INTEGUSEAL, a preoperative microbial skin sealant, on the rate of surgical site infections after cardiac surgery. Int J Infect Dis 15:395-400 
32. Eckardstein AS von, Lim CH, Dohmen PM et al (2011) A randomized trial of a skin sealant to reduce the risk of incision contamination in cardiac surgery. Ann Thorac Surg 92:632-637

33. Ecri Institute (2007) Cultures, bundles, and other options for MRSA control. Health Hazard Manage Monit 20:1-10

34. Edlich RF, Long WB, Gubler DK et al (2009) Dangers of cornstarch powder on medical gloves: seeking a solution. Ann Plast Surg 63:111-115

35. Ellis $H$ (2008) Evolution of the surgical glove. J Am Coll Surg 207:948-950

36. Flores-Maldonado A, Medina-Escobedo CE, RíosRodríguez HM, Fernández-Domínguez R (2011) Mild perioperative hypothermia and the risk of wound infection. Int J Evid Based Healthc 9:337345

37. Foca M, Jakob K, Whittier S et al (2000) Endemic Pseudomonas aeruginosa infection in a neonatal intensive care unit. N Engl J Med 343:695-700

38. Forrester BG, Roth VS (1998) Hand dermatitis in intensiv care units. J Occup Environ Med 40:881885

39. Fry DE (2011) Colon preparation and surgical site infection. Am J Surg 202:225-232

40. Gastmeier P, Geffers C, Rüden H et al (2003) Erläuterungen zu den Empfehlungen der Kommission für Krankenhaushygiene und Infektionsprävention zur Surveillance von postoperativen Wundinfektionen in Einrichtungen für das ambulante Operieren. Bundesgesundheitsblatt Gesundheitsforschung Gesundheitsschutz 46:765-769

41. Gastmeier P, Breier AC, Sohr D, Geffers C (2012) Prävention der postoperativen Wundinfektionen. Erkenntnisse aus 14 Jahren KISS (Krankenhausinfektionssurveillancesystem). Trauma Berufskrankh [Suppl 2] 14:110-111

42. Gordin FM, Schultz ME, Huber R et al (2007) A cluster of hemodialysis-related bacteremia linked to artificial fingernails. Inf Contr Hosp Epidemiol 28:743-744

43. Große-Schütte K, Assadian O, Hübner NO et al (2011) Practices of skin care among nurses in medical and surgical intensive care units: results of a self-administered questionnaire. GMS Krankenhhyg Interdiszip 6:Doc08

44. Gupta A, Della-Latta P, Todd B et al (2004) Outbreak of extended-spectrum beta-lactamaseproducing Klebsiella pneumoniae in a neonatal intensive care unit linked to artificial nails. Infect Control Hosp Epidemiol 25:210-215

45. Harnoss JC, Partecke LI, Heidecke CD et al (2010) Concentration of bacteria passing through puncture holes in surgical gloves. Am J Infect Control 38:154-158

46. Haynes AB, Weiser TG, Berry WR et al (2009) A surgical safety checklist to reduce morbidity and mortality in a global population. N Engl J Med 360:491-499

47. Hedderwick SA, McNeil SA, Lyons MJ, Kauffman CA (2000) Pathogenic organisms associated with artificial fingernails worn by healthcare workers. Infect Control Hosp Epidemiol 21:505-509

48. Heeg P, Christiansen B (1993) Hautantiseptik. In: Kramer A, Gröschel D, Heeg P et al (Hrsg) Klinische Antiseptik. Springer, Berlin Heidelberg New York, S 105-119

49. Heudorf U (2011) Zehn Jahre Infektionsschutzgesetz: Hygiene beim ambulanten Operieren in der Arztpraxis - Daten des Amtes für Gesundheit in Frankfurt am Main. Hyg Med 36:202-209

50. Hübner NO, Kellner NB, Partecke U et al (2011) Determination of antiseptic efficacy of rubs on the forearm and consequences for surgical hand disinfection. J Hosp Infect 78:11-15
51. Hübner NO, Fleßa S, Jakisch R et al (2012) Review of indicators for cross-sectoral optimization of nosocomial infection prophylaxis - a perspective from structurally- and process-oriented hygiene. GMS Krankenhhyg Interdiszip 7:Doc15

52. International Organization for Standardization (ISO) (1996) ISO 13488. Quality systems - Medical devices - Particular requirements for the application of ISO 9002. International Organization for Standardization (ISO), Genf

53. International Organization for Standardization (ISO) (2003) DIN EN ISO 13485. Medical devices quality management systems - requirements for regulatory purposes. International Organization for Standardization (ISO), Genf

54. Ito M, Sugito M, Kobayashi A et al (2009) Influence of learning curve on short-term results after laparoscopic resection for rectal cancer. Surg Endosc 23:403-408

55. Iyer A, Gilfillan I, Thakur S, Sharma S (2011) Reduction of surgical site infection using a microbial sealant: a randomized trial. J Thorax Cardiovasc Surg 142:438-442

56. Juaneda I, Moser F, Eynard H et al (2008) Granulomatous peritonitis due to the starch used in surgical gloves. Medicina (B Aires) 68:222-224

57. Kampf G, Ostermeyer C (2009) A 1-minute hand wash does not impair the efficacy of a propanolbased hand rub in two consecutive surgical hand disinfection procedures. Eur J Clin Microbiol Infect Dis 28:1357-1362

58. Kampf G, Ostermeyer C, Kohlmann T (2008) Bacterial population kinetics on hands during 2 consecutive surgical hand disinfection procedures. Am J Infect Control 36:369-374

59. Kikuchi-Numagami K, Saishu T, Fukaya M et al (1999) Irritancy of scrubbing up for surgery with or without a brush. Acta Derm Venereol 79:230 232

60. Kramer A (2013) Maßnahmen zur Vermeidung der postoperativen Wundinfektion. Trauma Berufskrankh [Suppl 2] 15:129-133

61. Kramer A, Jünger M, Kampf G (2005) Hygienische und dermatologische Aspekte der Händedesinfektion und der prophylaktischen Hautantiseptik. Hautarzt 56:743-751

62. Kramer A, Assadian O, Gruber B, Lademann J (2008) Prävention von postoperativen Wundinfektionen, Teil 1: Präoperative Maßnahmen - Einfluss der Haarentfernung. Hyg Med 33:402-407

63. Kramer A, Hübner N, Below H et al (2008) Improving adherence to surgical hand preparation. J Hosp Infect [Suppl 1] 70:35-43

64. Kramer A, Assadian O, Lademann J (2010) Prävention postoperativer Wundinfektionen durch Abkleben des OP-Felds mit lod-imprägnierter Inzisionsfolie (loban ${ }^{\circledR} 2$ ). GMS Hyg Infect Contr 5:Doc08

65. Kramer A, Schilling M, Heidecke CD (2010) Infektionspräventions-Check-in und Infektionspräventions-Check-out zur Prävention nosokomialer Infektionen. Zentralbl Chir 135:44-48

66. Kramer A, Assadian O, Wendt M et al (2011) Functional separation of septic and aseptic surgical procedures. GMS Krankenhhyg Interdiszip 6:Doc12

67. Kramer A, Assadian O, Mittelmeier W, Krüger CM (2012) Hygiene in der Orthopädie und Unfallchirurgie. Schwerpunkte der Infektionsprävention in Klinik und Praxis. Orthop Unfallchir Up2date 7:315-333

68. Kramer A, Assadian O, Helfrich J et al (2013) Umsetzung des Infektionsschutzgesetzes durch die Landeshygieneverordnungen. Hyg Med 38:427432
69. Kramer A, Henschel W, Wegner C, Heidecke CD (2013) Prospektive Studie zum dermatologischen Nutzen von Hautschutz und Hautpflege bei einem chirurgischen Team (Abstract). 10. Ulmer Symposium Krankenhausinfektionen Epidemiologie Hygienemaßnahmen Antibiotikatherapie, 19.-22.03.2013, Ulm. http://www.uniklinik-ulm de/fileadmin/Institute/Mikrobiologie_Immunologie/Mikrobiologie/Hygienesymposium/Dateien/Abstractband2013_ev_screen.pdf. Zugegriffen: 26.02 .2014

70. KRINKO (1997) Anhang zur Anlage zu Ziffern 5.1 und 4.3.3. Anforderungen der Hygiene beim ambulanten Operieren in Krankenhaus und Praxis. Bundesgesundheitsblatt 40:361-365

71. KRINKO (1999) Empfehlung zur Prävention und Kontrolle von methicillinresistenten Staphylococcus aureus-Stämmen (MRSA) in Krankenhäusern und anderen medizinischen Einrichtungen. Mitteilung der Kommission für Krankenhaushygiene und Infektionsprävention am RKI. Bundesgesundheitsblatt Gesundheitsforschung Gesundheitsschutz 42:954-958

72. KRINKO (2000) Anforderungen der Hygiene bei Operationen und anderen invasiven Eingriffen. Empfehlung der Kommission für Krankenhaushygiene und Infektionsprävention. Bundesgesundheitsblatt Gesundheitsforschung Gesundheitsschutz 43:644-648

73. KRINKO (2000) Händehygiene. Empfehlung der Kommission für Krankenhaushygiene und Infektionsprävention. Bundesgesundheitsblatt Gesundheitsforschung Gesundheitsschutz 43:230233

74. KRINKO (2001) Mitteilung der Kommission für Krankenhaushygiene und Infektionsprävention zur Surveillance (Erfassung und Bewertung) von nosokomialen Infektionen (Umsetzung von § 23 IfSG). Vorwort des Robert Koch-Instituts zur Empfehlung der Kommission für Krankenhaushygiene und Infektionsprävention zur Surveillance (Erfassung und Bewertung) von nosokomialen Infektionen. Bundesgesundheitsblatt Gesundheitsforschung Gesundheitsschutz 44:523-536

75. KRINKO (2003) Surveillance von postoperativen Wundinfektionen in Einrichtungen für das ambulante Operieren gemäß § 23 Abs. 1 IfSG. Empfehlung der Kommission für Krankenhaushygiene und Infektionsprävention. Bundesgesundheitsblatt Gesundheitsforschung Gesundheitsschutz 46:791-795

76. KRINKO (2004) Anforderungen an die Hygiene bei der Reinigung und Desinfektion von Flächen. Empfehlung der Kommission für Krankenhaushygiene und Infektionsprävention. Bundesgesundheitsblatt Gesundheitsforschung Gesundheitsschutz 47:51-61

77. KRINKO (2007) Prävention postoperativer Infektionen im Operationsgebiet. Empfehlung der Kommission für Krankenhaushygiene und Infektionsprävention. Bundesgesundheitsblatt Gesundheitsforschung Gesundheitsschutz 50:377393

78. KRINKO (2009) Personelle und organisatorische Voraussetzungen zur Prävention nosokomialer Infektionen. Empfehlung der Kommission für Krankenhaushygiene und Infektionsprävention. Bundesgesundheitsblatt 52:951-962

79. KRINKO (2010) Kommentar der KRINKO zur DIN 1946-4 (2008), Raumlufttechnische Anlagen. Epidemiol Bull 4:35

80. KRINKO (2010) Die Kategorien in der Richtlinie für Krankenhaushygiene und Infektionsprävention - Aktualisierung der Definitionen. Bundesgesundheitsblatt 53:754-756 
81. KRINKO (2012) Anforderungen an die Hygiene bei der Aufbereitung von Medizinprodukten Empfehlung der Kommission für Krankenhaushygiene und Infektionsprävention (KRINKO) beim Robert Koch-Institut (RKI) und des Bundesinstitutes für Arzneimittel und Medizinprodukte (BfArM). Bundesgesundheitsblatt 55:1244-1310

82. KRINKO (2012) Hygienemaßnahmen bei Infektionen oder Besiedlung mit multiresistenten gramnegativen Stäbchen. Empfehlung der Kommission für Krankenhaushygiene und Infektionsprävention (KRINKO) beim Robert Koch-Institut (RKI). Bundesgesundheitsblatt 55:1311-1354

83. Lammers T (1978) Zur Prüfung der Händedesinfektion. Hyg Med 3:316-318

84. Lavu H, Klinge MJ, Nowcid LJ et al (2012) Perioperative surgical care bundle reduces pancreatico-duodenectomy wound infections. J Surg Res 174:215-221

85. Lehtinen SJ, Onicescu G, Kuhn KM et al (2010) Normothermia to prevent surgical site infections after gastrointestinal surgery: holy grail or false idol? Ann Surg 252:696-704

86. Louis C (2011) Ansell healthcare receives CE mark for GAMMEX ${ }^{\circledR}$ powder-free gloves with AMT antimicrobial technology. Ansell, Brüssel. http:// www.anselleurope.com/ansell/ansell-healthcarenews/ansell-healthcare-press-releases-1/index. cfm?!=11613. Zugegriffen: 26.02 .2014

87. Mäkela P (1993) Gesunde Haut als Voraussetzung für eine effektive Händedesinfektion. In: Kramer A, Gröschel D, Heeg P et al (Hrsg) Klinische Antiseptik. Springer, Berlin Heidelberg New York, S 97-103

88. Mangram AJ, Horan TC, Pearson ML et al (1999) The Hospital Infection Control Practices Advisory Committee. Guideline for prevention of surgical site infection, 1999. Infect Control Hosp Epidemiol 20:247-278

89. Matsou A, Vrakas G, Doulgerakis M et al (2011) Mechanical bowel preparation before elective colorectal surgery: is it necessary? Tech Coloproctol [Suppl 1] 15:S59-S62

90. McHugh SM, Hill ADK, Humphreys H (2011) Intraoperative technique as a factor in the prevention of surgical site infection. J Hosp Infect 78:1-4

91. McNeil SA, Foster CL, Hedderwick SA, Kauffman CA (2001) Effect of hand cleansing with antimicrobial soap or alcohol-based gel on microbial colonization of artificial fingernails worn by health care workers. Clin Infect Dis 32:367-372

92. Melling AC, Ali B, Scott EM, Leaper DJ (2001) Effects of preoperative warming on the incidence of wound infection after clean surgery: a randomised controlled trial. Arch Med Res 32:227-231

93. Möckel D (2014) Prävention postoperativer Wundinfektionen mit Hilfe des Infektionspräventions-Check-out für Patienten. Med. Dissertation, Universität Greifswald

94. Molina-Cabrillana J, Chirino Cabrera A, Rodriguez-Alvarez JP et al (2007) Effect of surveillance on surgical site infection rate in knee and hip arthroplasty. Rev Clin Esp 207:388-393

95. Moola S, Lockwood C (2013) Effectiveness of strategies for the management and/or prevention of hypothermia within the adult perioperative environment. Br J Surg 100:465-473

96. Moolenaar RL, Crutcher JM, San Joaquin VH et al (2000) A prolonged outbreak of Pseudomonas aeruginosa in a neonatal intensive care unit: did staff fingernails play a role in disease transmission? Infect Control Hosp Epidemiol 21:80-85

97. Murdoch H, Taylor D, Dickinson J et al (2006) Surface decontamination of surgical instruments: an ongoing dilemma. J Hosp Infect 63:432-438
98. Murray BW, Huerta S, Dineen S, Anthony T (2010) Surgical site infection in colorectal surgery. J Am Coll Surg 211:812-822

99. Nicolai P, Aldam CH, Allen PW (1997) Increased awareness of glove perforation in major joint replacement. A prospective, randomised study of Regent Biogel Reveal gloves. J Bone Joint Surg Br 79:371-373

100. Osman MO, Jensen SL (1999) Surgical gloves: current problems. World J Surg 23:630-637

101. Parks PJ, Babadjanian C, Johnson EJ, Walters SA (2007) Infection reduction with antimicrobial-impregnated incise foils. European Bone and Joint Infection Society, 26th annual meeting, Corfu, Greece, 20-22 Sep 07. European Bone and Joint Infection Society, Wien

102. Parry MF, Grant B, Yukna M et al (2001) Candida osteomyelitis and diskitis after spinal surgery: an outbreak that implicates artificial nail use. Clin Infect Dis 32:352-357

103. Partecke LI, Goerdt AM, Langner I et al (2009) The incidence of micro-perforation in surgical gloves depends on duration of wearing. Infect Control Hosp Epidemiol 30:409-414

104. Passaro DJ, Waring L, Armstrong R et al (1997) Postoperative Serratia marcescens wound infections traced to an out-of-hospital source. J Infect Dis 175:992-995

105. Pitten FA, Müller P, Heeg P, Kramer A (1998/99) Untersuchungen zur wiederholten Desinfizierbarkeit von Einweghandschuhen während des Tragens. Zentralbl Hyg Umweltmed 201:555-562

106. Porteous J (2002) Artificial nails, very real risks. Can Oper Room Nurs J 20:16-17, 20-21

107. Pottinger J, Burns S, Manske C (1989) Bacterial carriage by artificial versus natural nails. Am J Infect Control 17:340-344

108. Rao N, Cannella B, Crossett LS et al (2011) Preoperative screening/decolonization for Staphylococcus aureus to prevent orthopedic surgical site infection: prospective cohort study with 2-year follow-up. J Arthroplasty 26:1501-1507

109. Rijen MM van, Bonten M, Wenzel RP, Kluytmans JA (2008) Intranasal mupirocin for reduction of Staphylococcus aureus infections in surgical patients with nasal carriage: a systematic review. J Antimicrob Chemother 61:254-261

110. Saiman L, Lerner A, Saal L et al (2002) Banning artificial nails from health care settings. Am J Infect Control 30:252-254

111. Scholz S (2013) Einfluss von Biosorb auf die Langzeitwirkung der chirurgischen Händedesinfektion und die Schweißproduktion der Hand. Med. Dissertation, Universität Greifswald

112. Shuman AG, Shuman EK, Hauff SJ et al (2012) Preoperative topical antimicrobial decolonization in head and neck surgery. Laryngoscope 122:2454-2460

113. Sjösten ACE, Ellis H, Edelstam GAB (2004) Retrograde migration of glove powder in the human female genital tract. Hum Reprod 19:991-995

114. Thiede B, Kramer A (2013) Evaluation of reprocessing medical devices in 14 German regional hospitals and at 27 medical practitioners' offices within the European context - consequences for European harmonization. GMS Hyg Infect Control 8:Doc20

115. Thimour-Bergström L, Roman-Emanuel C, Scherstén H et al (2012) Triclosan-coated sutures reduce surgical site infection after open vein harvesting in coronary artery bypass grafting patients: a randomized controlled trial. World J Surg $36: 2528-2534$
116. Thompson KM, Oldenburg WA, Deschamps C et al (2011) Chasing zero: the drive to eliminate surgical site infections. Ann Surg 254:430-437

117. Toles A (2002) Artificial nails: are they putting patients at risk? A review of the research. J Pediatr Oncol Nurs 19:164-171

118. Towfigh S, Cheadle WG, Lowry SF et al (2008) Significant reduction in incidence of wound contamination by skin flora through use of microbial sealant. Arch Surg 143:885-891

119. Trussell J, Gerkin R, Coates B et al (2008) Impact of a patient care pathway protocol on surgical site infection rates in cardiothoracic surgery patients. Am J Surg 196:883-889

120. Turtiainen J, Saimanen El, Mäkinen KT et al (2012) Effect of triclosan-coated sutures on the incidence of surgical wound infection after lower limb revascularization surgery: a randomized controlled trial. Ann Surg 255:854-859

121. Ulmer M, Lademann J, Patzelt $A$ et al (in press) New strategies for preoperative skin antisepsis. Skin Pharmacol Physiol in press

122. Vries JJ de, Baas WH, Ploeg K van der et al (2006) Outbreak of Serratia marcescens colonization and infection traced to a healthcare worker with long-term carriage on the hands. Infect Control Hosp Epidemiol 27:1153-1158

123. Waldow T, Szlapka M, Hensel J et al (2012) Skin sealant InteguSeal ${ }^{\circledR}$ has no impact on prevention of postoperative mediastinitis after cardiac surgery. J Hosp Infect 81:278-282

124. Wang ZX, Jiang CP, Cao Y, Ding YT (2013) Systematic review and meta-analysis of triclosan-coated sutures for the prevention of surgical-site infection. Eur J Cardiothorac Surg 44:931-938

125. Waterman TR, Smeak DD, Kowalski J, Hade EM (2006) Comparison of bacterial counts in glove juice of surgeons wearing smooth band rings versus those without rings. Am J Infect Control 34:421-425

126. Webster J, Alghamdi AA (2007) Use of plastic adhesive drapes during surgery for preventing surgical site infection. Cochrane Database Syst Rev 4:CD006353

127. Weimann A (2012) Infektionsprävention durch gezielte Ernährung und Probiotika. In: Kramer A, Assadian O, Exner M et al (Hrsg) Krankenhausund Praxishygiene. Elsevier Urban \& Fischer, München, S 288-290

128. Wilson SE (2008) Microbial sealing: a new approach to reducing contamination. J Hosp Infect [Suppl 2] 70:11-14

129. World Alliance for Patient Safety (2008) The second global patient safety challenge. Safe surgery saves lives. WHO, Genf. http://www.who. int/patientsafety/safesurgery/knowledge_base/SSSL_Brochure_finalJun08.pdf. Zugegriffen: 11.3.2014

130. Young B, Ng TM, Teng C et al (2011) Nonconcordance with surgical site infection prevention guidelines and rates of surgical site infections for general surgical, neurological, and orthopedic procedures. Antimicrob Agents Chemother 55:46594663

131. Young H, Knepper B, Vigil C et al (2013) Sustained reduction in surgical site infection after abdominal hysterectomy. Surg Infect (Larchmt) 14:460463 